\title{
STABILITY OF LIPSCHITZ-TYPE FUNCTIONS UNDER POINTWISE PRODUCT AND RECIPROCATION
}

\author{
GERALD BEER, LUIS C. GARCÍA-LIROLA, AND M. ISABEL GARRIDO
}

\begin{abstract}
This article provides necessary and sufficient conditions on the structure of a metric space such that for various vector lattices of real-valued Lipschitz-type functions defined on the metric space, the vector lattice is stable under pointwise product, and such that the reciprocal of each non-vanishing member of the vector lattice remains in the vector lattice. In each case the family of metric spaces for which the first property holds contains the family of metric spaces for which the second property holds. At the end we prove some extension theorems for classes of locally Lipschitz functions that complement known results for Cauchy continuous functions and for uniformly continuous functions.
\end{abstract}

\section{INTRODUCTION}

Let $\langle X, d\rangle$ and $\langle Y, \rho\rangle$ be metric spaces. A function $f: X \rightarrow Y$ is called Lipschitz if for some $\lambda>0$ and each $x_{1}, x_{2} \in X$ we have $\rho\left(f\left(x_{1}\right), f\left(x_{2}\right)\right) \leq \lambda d\left(x_{1}, x_{2}\right)$. For added precision, we can say that $f$ is $\lambda$-Lipschitz. The real-valued Lipschitz functions $\operatorname{Lip}(X)$ on $X$ form a vector lattice that contains the constant functions but which is not in general stable under pointwise products. However, if $f$ and $g$ are bounded Lipschitz functions when restricted to a subset $A$ of $X$, then their product so restricted is Lipschitz. In particular, this is true if $A$ is a bounded subset of the metric space. Furthermore, given a non-vanishing member $f$ of $\operatorname{Lip}(X)$, it may be true that $\frac{1}{f}$ is no longer Lipschitz. For example $f(x)=\frac{1}{1+x^{2}}$ having bounded derivative is a Lipschitz function on $\mathbb{R}$, whereas its reciprocal is not Lipschitz. We will say that a class $\Omega$ of real-valued functions on $\langle X, d\rangle$ is stable under reciprocation if whenever $f \in \Omega$ is never zero, then $\frac{1}{f} \in \Omega$ ensues.

Of course, $f: X \rightarrow Y$ is called locally Lipschitz if it is Lipschitz when restricted to some neighborhood of each point of the space. We denote the real-valued locally Lipschitz functions on $X$ by $L L(X)$. It is well known that a function is locally Lipschitz if and only if it is Lipschitz when restricted to each compact subset of $X$ (see, e.g., $[7,14,30])$. Since compact subsets are bounded, such functions are stable under pointwise product. Furthermore, if $f$ is Lipschitz on each compact subset and

Date: March 26, 2020.

2010 Mathematics Subject Classification. Primary 54E40, 26A16; Secondary 46E15, 54C30.

Key words and phrases. Lipschitz function, locally Lipschitz function, Lipschitz in the small function, Cauchy-Lipschitz function, pointwise product, reciprocation, UC-space, cofinal completeness, modulus of continuity.

The first author would like to thank Universidad Complutense for its hospitality in September 2019. The second author is partially supported by grants MTM2017-83262-C2-2-P and Fundación Séneca CARM 20906/PI/18. He is also supported by a postdoctoral grant from Fundación SénecaAgencia de Ciencia y Tecnología de la Región de Murcia (Spain). The third author was partially supported by DGES grant PGC2018-097286-B-I00 (Spain). 
non-vanishing, then $|f|$ when restricted to each compact subset attains a positive minimum value, whence the reciprocal of $f$ is Lipschitz on each compact subset. Thus the locally Lipschitz functions in general are stable under both pointwise product and reciprocation.

In this article we begin by showing that $\operatorname{Lip}(X)$ is stable under pointwise product if and only if $X$ is bounded, and stable under reciprocation if and only if $X$ is compact. The first fact is a standard exercise, and we view the second as a folktheorem for which we have no reference. We also look at the real-valued CauchyLipschitz functions and uniformly locally Lipschitz functions as studied by Beer and Garrido $[6,7]$ and the real-valued Lipschitz in the small functions as introduced by Luukkainen [25]. The analysis leads us to consider classes of metric spaces that may not be familiar to all readers: the cofinally complete metric spaces, the UC or Atsuji metric spaces, and a class of metric spaces introduced recently by J. CabelloSánchez [13] characterized by the stability of the uniformly continuous real-valued functions on $X$ under pointwise product.

For real-valued functions, each class of Lipschitz-type functions forms a vector lattice containing the constant functions (where the pointwise order is understood). In this setting, the metric spaces for which the class is stable under pointwise product must include the spaces for which the class is stable under reciprocation. We confirm this assertion, in which metric structure plays no role, presently. A vector lattice of real-valued functions containing the constants that is stable under pointwise product forms a ring with multiplicative identity.

To show that a vector space $V$ of real-valued functions is stable under pointwise product, it suffices to show that $f^{2} \in V$ whenever $f \in V$, in view of the identity

$$
f g=\frac{1}{4}\left((f+g)^{2}-(f-g)^{2}\right) .
$$

Theorem 1.1. Let $V$ be a vector lattice of real-valued functions on a set $X$ containing the constant functions. If $V$ is stable under reciprocation, then $V$ is stable under pointwise product.

Proof. We first remind the reader that a vector lattice of functions is stable under taking the absolute value of its members because $|f|=\max \{f, 0\}-\min \{f, 0\}$.

We first claim that if $g \in V$ and there exists $\alpha>0$ such that for all $x \in$ $X,|g(x)| \neq \alpha$, then $g^{2} \in V$. Indeed, note that

$$
\frac{1}{g^{2}-\alpha^{2}}=\frac{1}{2 \alpha(g-\alpha)}-\frac{1}{2 \alpha(g+\alpha)}
$$

from which $g^{2}-\alpha^{2}$ and then $g^{2}$ belong to $V$.

Now let $f \in V$ be arbitrary and put $g:=|f|+2$ which fulfills the above condition with $\alpha=1$. Then $g^{2} \in V$ and $f^{2}=g^{2}-4|f|-4$. This means that $f^{2} \in V$ as well.

Corollary 1.2. Let $V$ be a vector lattice of real-valued functions on a set $X$ containing the constant functions. If $V$ is stable under reciprocation, then whenever $f \in V$ is non-vanishing and $g \in V$, we have $\frac{g}{f} \in V$.

In the last section of this article, we prove some extension theorems for CauchyLipschitz functions and for Lipschitz in the small functions anticipated by established extension results for Cauchy continuous functions and for uniformly continuous functions. In the process, we give necessary and sufficient conditions for a 
Lipschitz in the small function between metric spaces to be Lipschitz in terms of its modulus of continuity function.

\section{Preliminaries}

All metric spaces are assumed to contain at least two points. We denote the set of limit points of the space $\langle X, d\rangle$ by $X^{\prime}$. We write $S_{d}(x, \varepsilon)$ for the open ball of center $x$ and radius $\varepsilon>0$ in $X$. We write $S_{d}(A, \varepsilon)$ for $\cup_{a \in A} S_{d}(a, \varepsilon)$, and call this set the $\varepsilon$-enlargement of $A$ [2]. For $x \in X$ and $A$ a nonempty subset of $\langle X, d\rangle$, we put $d(x, A):=\inf \{d(x, a): a \in A\}$. We define the isolation functional $I_{d}$ for $\langle X, d\rangle$ by the formula $I_{d}(x):=d(x, X \backslash\{x\})$. Of course $I_{d}(x)=0$ means that $x \in X^{\prime}$. All distance functionals and isolation functionals are 1-Lipschitz.

We denote the usual $d$-diameter of a nonempty subset $A$ of $X$ by $\operatorname{diam}_{d}(A)$. A subset $A$ of $X$ is called bounded if it is either empty or has finite diameter; equivalently, $A$ is a subset of some open ball in $X$. A subset $A$ of $X$ is called totally bounded if for each $\varepsilon>0$ there exists a finite subset $F$ of $X$ with $A \subseteq S_{d}(F, \varepsilon)$. It is called relatively compact if it has compact closure. A metric space is called boundedly compact if each bounded subset is relatively compact. Each relatively compact subset of $X$ is totally bounded, and the converse is true if and only if the metric $d$ is complete. If $A$ is a nonempty subset of $X$ and $\varepsilon>0$, we define $S_{d}^{n}(A, \varepsilon)$ recursively by $S_{d}^{1}(A, \varepsilon)=S_{d}(A, \varepsilon)$ and $S_{d}^{n+1}(A, \varepsilon)=S_{d}\left(S_{d}^{n}(A, \varepsilon), \varepsilon\right)$. A subset $A$ of $X$ is called Bourbaki bounded [5, 18] if for every $\varepsilon>0$ there exists a nonempty finite subset $F$ of $X$ and $n \in \mathbb{N}$ with $A \subseteq S_{d}^{n}(F, \varepsilon)$. Each totally bounded subset is Bourbaki bounded and each Bourbaki bounded subset is metrically bounded. Each relatively compact set is Bourbaki bounded as each relatively compact set is totally bounded. A nonempty subset $A$ of a metric space $\langle X, d\rangle$ is Bourbaki bounded if and only each uniformly continuous real-valued function on $X$ is bounded when restricted to $A[5,19]$.

As a subset of a metric space is (a) relatively compact if and only if each sequence in it has a convergent subsequence, and (b) totally bounded if and only if each sequence in it has a Cauchy subsequence, a subset is Bourbaki bounded if and only if each sequence in it has a Bourbaki-Cauchy subsequence [18, Theorem 4], as we now define, in a way paralleling the standard definition of Cauchy sequence.

Definition 2.1. A sequence $\left\langle x_{n}\right\rangle$ in a metric space $\langle X, d\rangle$ is called Bourbaki-Cauchy provided for each $\varepsilon>0$ there exists $k \in \mathbb{N}$ and $m \in \mathbb{N}$ such that whenever $n$ and $j$ exceed $k$, we have $x_{n} \in S_{d}^{m}\left(\left\{x_{j}\right\}, \varepsilon\right)$.

A metric space is called Bourbaki complete provided each Bourbaki-Cauchy sequence in it clusters [18]. Obviously, Bourbaki completeness implies completeness of the metric as it usually understood. A metric space is Bourbaki complete if and only if each Bourbaki bounded subset is relatively compact.

The metrically bounded subsets, the relatively compact subsets, the totally bounded subsets, and the Bourbaki bounded subsets each form a bornology on the underlying metric space: (1) each contains the singletons; (2) each is a hereditary family of subsets; (3) each is stable under finite unions.

We call a function from $\langle X, d\rangle$ to $\langle Y, \rho\rangle$ Cauchy continuous $[7,11,31]$ if it maps Cauchy sequences in $X$ to Cauchy sequences in $Y$. Each Cauchy continuous function is continuous, and each uniformly continuous function is Cauchy continuous. It is notable that each Cauchy continuous real-valued function defined on a nonempty 
subset of $\langle X, d\rangle$ can be extended to a globally defined Cauchy continuous function [31]. For this reason alone, the class of Cauchy continuous functions deserves some coverage in analysis texts.

We now formally introduce some subclasses of the locally Lipschitz functions in order of decreasing size.

- $f: X \rightarrow Y$ is called Cauchy-Lipschitz if its restriction to the range of each Cauchy sequence in $X$ is Lipschitz;

- $f: X \rightarrow Y$ is called uniformly locally Lipschitz if for some $\varepsilon>0$ the restriction of $f$ to each open ball of radius $\varepsilon$ is Lipschitz;

- $f: X \rightarrow Y$ is called Lipschitz in the small if there exists $\lambda>0$ and $\delta>0$ such that $d\left(x_{1}, x_{2}\right)<\delta \Rightarrow \rho\left(f\left(x_{1}\right), f\left(x_{2}\right)\right) \leq \lambda d\left(x_{1}, x_{2}\right)$.

In the case of real-valued functions, each of these classes forms a vector lattice containing the constants. In this setting, we denote these vector lattices by $\mathrm{CL}(X), U L L(X)$ and $L S(X)$, respectively. Pairwise coincidence of our four classes of locally Lipschitz functions has been determined [6, 7]. Each locally Lipschitz function is continuous; moreover, $L L(X)$ is uniformly dense in the continuous realvalued functions $[7,14,16,26]$; each Cauchy-Lipschitz function is Cauchy continuous and $\mathrm{CL}(X)$ is uniformly dense in the Cauchy continuous real-valued functions [7]; each Lipschitz in the small function is uniformly continuous and $L S(X)$ is uniformly dense in the uniformly continuous real-valued functions [6, 17]. A function is Cauchy-Lipschitz if and only if its restriction to each totally bounded subset is Lipschitz [7, Proposition 3.4]. Those subsets of a metric space $\langle X, d\rangle$ on which each Lipschitz in the small function is Lipschitz when restricted to them - called the small determined subsets - have been internally characterized by Leung and Tang [24], who also noted that this feature of the subset is determined by real-valued Lipschitz in the small functions. Unfortunately a function can be Lipschitz on each such subset without being actually Lipschitz in the small [8]. We note that a bounded subset of $X$ is small determined if and only if it is Bourbaki bounded [6, Theorem 4.4]. Metric spaces that are small determined were first considered by Garrido and Jaramillo [17].

We close this section with a lemma that we employ going forward.

Lemma 2.2. Let $A$ be a nonempty closed subset of $\langle X, d\rangle$ and let $f: A \rightarrow(0, \infty)$ be $\lambda$-Lipschitz for some positive $\lambda$. Then $f$ has a strictly positive $\lambda$-Lipschitz extension to $X$.

Proof. As is well known [20, pp. 43-44], $x \mapsto \inf _{a \in A} f(a)+\lambda d(x, a)$ is a $\lambda$-Lipschitz extension of $f$ to the entire space, and it is easily verified that this extension majorizes all others. For $x \notin A$, we have for each $a \in A$

$$
0<\lambda d(x, A) \leq f(a)+\lambda d(x, a)
$$

because $A$ is closed. As a result, the extension has strictly positive values.

\section{LipsChitz FUnCTIONS}

Necessary and sufficient conditions for stability under pointwise product for realvalued Lipschitz functions are transparent. We obtain these as a special case of a more generally applicable result. 
Proposition 3.1. Let $\mathcal{A}$ be a family of nonempty subsets of a metric space $\langle X, d\rangle$ and let $\mathfrak{F}$ be the family of real-valued functions that are Lipschitz when restricted to each member of $\mathcal{A}$. Then $\mathfrak{F}$ is stable under pointwise product if and only if each member of $\mathcal{A}$ is bounded.

Proof. Sufficiency is obvious. For necessity, suppose some $A \in \mathcal{A}$ is unbounded. Fixing $a_{0} \in A$, while $f(x)=d\left(x, a_{0}\right)(x \in X)$ is 1-Lipschitz when restricted to each member of $\mathcal{A}, f^{2}$ fails to be Lipschitz when restricted to $A$.

Letting $\mathcal{A}=\{X\}$ in Proposition 3.1, we immediately obtain

Theorem 3.2. Let $\langle X, d\rangle$ be a metric space. Then $\operatorname{Lip}(X)$ is stable under pointwise product if and only if $d$ is a bounded metric.

Turning to reciprocation, we will use the following lemma.

Lemma 3.3. Let $\langle X, d\rangle$ be a bounded metric space. Assume that $\frac{1}{f} \in \operatorname{Lip}(X)$ whenever $f$ is a non-vanishing Lipschitz function on $X$. Then every Lipschitz function on $X$ attains its infimum.

Proof. Let $f \in \operatorname{Lip}(X)$; since $X$ is bounded, $\alpha:=\inf _{x \in X} f(x)$ is finite. Suppose $f$ does not attain its infimum. By replacing $f$ by $f-\alpha$, we may assume $\alpha=0$. Then $\frac{1}{f}$ would be an unbounded Lipschitz function defined on a bounded metric space which is impossible.

Theorem 3.4. Let $\langle X, d\rangle$ be a metric space. Then $\operatorname{Lip}(X)$ is stable under reciprocation if and only if $X$ is compact.

Proof. Sufficiency follows easily from the fact that for each non-vanishing $f \in$ $\operatorname{Lip}(X),|f|$ has an absolute minimum value. We turn to necessity. If $\operatorname{Lip}(X)$ is stable under reciprocation, by Theorem 1.1 and Theorem 3.2, $X$ must be bounded. It suffices to show for compactness that $X$ is countably compact [32, p. 125]. To this end, let $\left\{U_{n}: n \in \mathbb{N}\right\}$ be a countable open cover of $X$ and suppose that it does not admit a finite subcover. We may assume that $U_{n} \subseteq U_{n+1}$ for each $n$. Consider the 1-Lipschitz function

$$
f:=\sum_{n=1}^{\infty} \frac{1}{2^{n}} d\left(\cdot, X \backslash U_{n}\right)
$$

Putting $D:=\operatorname{diam}_{d}(X)$, we see that for all $x \in X, 0<f(x) \leq D$ because $\left\{U_{n}: n \in \mathbb{N}\right\}$ covers $X$. Moreover, as there is no finite subcover, for each $n \in \mathbb{N}$ there is $x_{n} \in X \backslash U_{n}$. We compute

$$
f\left(x_{n}\right)=\sum_{i=n+1}^{\infty} \frac{1}{2^{i}} d\left(x_{n}, X \backslash U_{i}\right) \leq \frac{D}{2^{n}} .
$$

Thus, the infimum of $f$ is zero, and it is not attained, in violation of Lemma 3.3.

Proposition 3.5. Let $\mathcal{A}$ be a family of nonempty subsets of a metric space $\langle X, d\rangle$ and let $\mathfrak{F}$ be the family of continuous real-valued functions that are Lipschitz when restricted to each member of $\mathcal{A}$.

(a) $\mathfrak{F}$ is stable under reciprocation if and only if every member of $\mathcal{A}$ is relatively compact. 
(b) In the case that $\mathcal{A}$ contains each nonempty relatively compact subset, then $\mathfrak{F}$ is stable under reciprocation if and only if $\mathfrak{F}=L L(X)$.

Proof. We first look at statement (a). For sufficiency, let $f \in \mathfrak{F}$ be nonvanishing and let $A \in \mathcal{A}$. By assumption there exists $\lambda>0$ such that $\left.f\right|_{A}$ is $\lambda$-Lipschitz. By continuity, $\left.f\right|_{\mathrm{cl}(A)}$ is also $\lambda$-Lipschitz, so by Theorem $3.4, \frac{1}{f}$ is Lipschitz on $\operatorname{cl}(A)$ and thus on $A$. For necessity, suppose $A \in \mathcal{A}$ is not relatively compact. The proof of Theorem 3.4 shows that there is a positive Lipschitz function $f$ on $\operatorname{cl}(A)$ whose reciprocal is not Lipschitz on $\operatorname{cl}(A)$ and therefore is not Lipschitz on $A$ by continuity. By Lemma 2.2, $f$ has a positive Lipschitz-constant preserving extension $g$ to $X$, and of course, $g \in \mathfrak{F}$. But $\frac{1}{g}$ cannot be in $\mathfrak{F}$ as its restriction to $A$ agrees with $\frac{1}{f}$.

For statement (b), if $\mathfrak{F}=L L(X)$, then clearly $\mathfrak{F}$ is stable under reciprocation. Conversely, if each nonempty relatively compact subset belongs to $\mathcal{A}$ and $\mathfrak{F}$ is stable under reciprocation, then by statement (a) $\mathcal{A}$ coincides with the nonempty relatively compact subsets, and as is well-known [30], this gives $\mathfrak{F}=L L(X)$.

In statement (b), the condition that $\mathcal{A}$ contains the relatively compact sets is equivalent to requiring that each member of $\mathfrak{F}$ is locally Lipschitz. We next give four applications of Proposition 3.1 and Proposition 3.5.

Theorem 3.6. The family of Cauchy-Lipschitz functions on a metric space $\langle X, d\rangle$ is always stable under pointwise product, and is stable under reciprocation if and only if $d$ is a complete metric.

Proof. Recall that $f: X \rightarrow \mathbb{R}$ is Cauchy-Lipschitz if and only if it is Lipschitz when restricted to each nonempty totally bounded subset [7, Proposition 3.4]. Since totally bounded sets are metrically bounded, by Proposition 3.1, the pointwise product of two Cauchy-Lipschitz functions is again Cauchy-Lipschitz with no restrictions on the metric space.

We turn to reciprocation. Since each relatively compact subset is totally bounded, by Proposition 3.5 (b), stability under reciprocation occurs provided the CauchyLipschitz real-valued functions coincide with the locally Lipschitz real-valued functions. This happens exactly when $d$ is a complete metric [7, Theorem 3.5].

Theorem 3.7. Let $\mathfrak{F}$ be the family of real-valued functions on a metric space $\langle X, d\rangle$ that are Lipschitz when restricted to each Bourbaki bounded subset of $X$. Then $\mathfrak{F}$ is always stable under pointwise product, and is stable under reciprocation if and only if $\langle X, d\rangle$ is Bourbaki complete.

Proof. Members of $\mathfrak{F}$ are Lipschitz restricted to each nonempty relatively compact subset and thus are continuous on $X$. Stability under pointwise product with no restriction again follows from Proposition 3.1. From [18, Theorem 9], each Bourbaki bounded subset is relatively compact if and only if the space is Bourbaki complete. Proposition 3.5(a) yields the stated criterion for stability under reciprocation.

In [8], it is shown that a function is Lipschitz when restricted to each Bourbaki bounded subset of $\langle X, d\rangle$ if and only if it is Lipschitz when restricted to the range of each Bourbaki-Cauchy sequence. It is also shown in [8] that the functions that are Lipschitz when restricted to each nonempty Bourbaki bounded subset coincide with $L L(X)$ if and only if $\langle X, d\rangle$ is Bourbaki complete, allowing us to alternatively use Proposition 3.5(b) in the proof of our last result. 
STABILITY OF LIPSCHITZ-TYPE FUNCTIONS UNDER POINTWISE PRODUCT AND RECIPROCATION

Theorem 3.8. Let $\mathfrak{F}$ be the family of real-valued functions on a metric space $\langle X, d\rangle$ that are Lipschitz when restricted to each small determined subset of $X$.

(a) $\mathfrak{F}$ is stable under pointwise product if and only if each small determined subset is Bourbaki bounded;

(b) $\mathfrak{F}$ is stable under reciprocation if and only if each small determined subset is Bourbaki bounded and $\langle X, d\rangle$ is Bourbaki complete.

Proof. The first statement follows from Proposition 3.1 and [6, Theorem 4.4]. The second statement follows from the first statement, Theorem 1.1, Proposition 3.5(a) and $[18$, Theorem 9].

We leave the simple proof of this final consequence to the reader.

Theorem 3.9. Let $\mathfrak{F}$ be the family of real-valued functions on a metric space that are Lipschitz when restricted to each bounded subset of $\langle X, d\rangle$. Then $\mathfrak{F}$ is always stable under pointwise product, and is stable under reciprocation if and only if $\langle X, d\rangle$ is boundedly compact.

\section{UNIFORMLY LOCALLY LIPSCHITZ FUNCTIONS}

We cannot use Proposition 3.1 and Proposition 3.5 to determine stability under pointwise product and reciprocation for $U L L(X)$ because in an arbitrary metric space, $U L L(X)$ cannot be described as the family of real-valued functions that are Lipschitz when restricted to a prescribed family of nonempty subsets $\mathcal{A}$ of the metric space.

Example 4.1. Consider the metric space $\langle X, d\rangle$ where $X=\mathbb{N}^{2}$ and $d$ is given by $d((n, k),(m, l))=1$ if $k \neq l$ and $d((n, k),(m, k))=\frac{1}{k}$ if $n \neq m$. Assume $\mathcal{A}$ were a family of subsets of $\mathbb{N}^{2}$ such that $U L L(X)$ coincides with the set of real-valued functions that are Lipschitz when restricted to each member of $\mathcal{A}$. We intend to show that $\mathcal{A}$ is made up of finite sets. Let $A \in \mathcal{A}$, and consider

$$
I:=\{k \in \mathbb{N}:(n, k) \in A \text { for some } n \in \mathbb{N}\} .
$$

Define $f: X \rightarrow \mathbb{R}$ by $f(n, k)=k$. As $f$ is constant on each ball of radius $\frac{1}{2}$, we have $f \in U L L(X)$. Now if $I$ were infinite, there would be a strictly increasing sequence $\left\langle k_{i}\right\rangle$ in $I$ and for each $i$ a positive integer $n_{i}$ such that $\left(n_{i}, k_{i}\right) \in A$. We have for $i>1$

$$
f\left(n_{i}, k_{i}\right)-f\left(n_{1}, k_{1}\right)=k_{i}-k_{1}=\left(k_{i}-k_{1}\right) d\left(\left(n_{i}, k_{i}\right),\left(n_{1}, k_{1}\right)\right),
$$

which shows that $\left.f\right|_{A}$ is not Lipschitz, a contradiction. We conclude that $I$ is finite.

Now fix $k$ in our finite set $I$. Define the function $g_{k}: X \rightarrow \mathbb{R}$ by $g_{k}(n, k)=n$ and $g_{k}(n, l)=0$ if $l \neq k$. Then $g_{k}$ is constant on each ball of radius $\frac{1}{2 k}$ and so $g_{k} \in U L L(X)$. This implies that $\left.g_{k}\right|_{A}$ is Lipschitz, and it follows that the set $A_{k}:=\{n \in \mathbb{N}:(n, k) \in A\}$ is finite because its points are equidistant. This shows that $A=\cup_{k \in I} A_{k}$ is also finite.

Finally, $h: X \rightarrow \mathbb{R}$ defined by $h(n, k)=n$ evidently is not uniformly locally Lipschitz. However $h$ is Lipschitz when restricted to each finite subset as is any real-valued function defined on $X$. That is a contradiction.

We note that the functions $f$ and $g_{k}$ defined above are actually Lipschitz in the small and so our construction also serves to show that $L S(X)$ cannot in general 
be described as a class of functions that are Lipschitz when restricted to certain subsets, complementing the very different counterexample provided in [8].

Theorem 4.2. Let $\langle X, d\rangle$ be a metric space. Then $U L L(X)$ is always stable under pointwise product.

Proof. Let $f$ and $g$ be uniformly locally Lipschitz real-valued functions on $X$. Then there exist $\delta_{1}>0$ and $\delta_{2}>0$ such that $f$ restricted to each open ball of radius $\delta_{1}$ is Lipschitz and $g$ restricted to each open ball of radius $\delta_{2}$ is Lipschitz. Put $\delta=\min \left\{\delta_{1}, \delta_{2}\right\}$; then $f$ and $g$ are both Lipschitz and bounded on each open ball of radius $\delta$ so that $f g$ is Lipschitz on each such ball.

We now turn to stability of $U L L(X)$ under reciprocation. A sequence $\left\langle x_{n}\right\rangle$ in a metric space $\langle X, d\rangle$ is called cofinally Cauchy if for each $\varepsilon>0$ there exists an infinite subset $\mathbb{N}_{\varepsilon}$ of $\mathbb{N}$ such that whenever $\{j, k\} \subseteq \mathbb{N}_{\varepsilon}$, we have $d\left(x_{j}, x_{k}\right)<\varepsilon$. The metric space is called cofinally complete provided each cofinally Cauchy sequence in $X$ clusters [3]. Net cofinal completeness in the context of uniform spaces is defined in the expected way [22], and the two notions for a metric space coincide.

Some important characterizations of cofinally completeness are the following:

- the space is uniformly paracompact [21, 28]: for each open cover $\mathcal{V}$ of $X$, there exists $\delta>0$ and an open refinement $\mathcal{U}$ of $\mathcal{V}$ such that for each $x \in$ $X, S_{d}(x, \delta)$ hits at most finitely many members of $\mathcal{U}$;

- $K:=\{x \in X: x$ has no compact neighborhood $\}$ is compact, and for each $\varepsilon>0$ there exists $\delta>0$ such that $x \notin S_{d}(K, \varepsilon) \Rightarrow\{w \in X: d(w, x) \leq \delta\}$ is compact [3];

- whenever $f: X \rightarrow \mathbb{R}$ is continuous, there exists $\delta>0$ such that $f$ restricted to each open ball of radius $\delta$ is bounded [3].

We note that the metric space of the initial example of this section was presented by Rice [28] to show that a locally compact metric space need not be cofinally complete.

Theorem 4.3. Let $\langle X, d\rangle$ be a metric space. The following conditions are equivalent:

(1) the metric space is cofinally complete;

(2) $U L L(X)$ is stable under reciprocation;

(3) whenever $g \in \operatorname{Lip}(X)$ is non-vanishing, we have $\frac{1}{g} \in U L L(X)$.

Proof. $(1) \Rightarrow(2)$. With cofinal completeness, $U L L(X)=L L(X)$ [6, Theorem 3.1], and the latter vector lattice is stable under reciprocation in general.

$(2) \Rightarrow(3)$. Since $\operatorname{Lip}(X) \subseteq U L L(X)$, this is trivial.

$(3) \Rightarrow(1)$. Suppose condition (1) fails. Then we can find a cofinally Cauchy sequence $\left\langle x_{n}\right\rangle$ in $X$ that does not cluster. In particular, the sequence has no constant subsequence. By passing to a subsequence, we may assume that $\left\langle x_{n}\right\rangle$ has distinct terms yet remains cofinally Cauchy. Without loss of generality, we may write $\mathbb{N}$ as a union of infinite subsets $\left\{\mathbb{M}_{j}: j \in \mathbb{N}\right\}$ such that whenever $\{i, k\} \subseteq \mathbb{M}_{j}$, we have $d\left(x_{i}, x_{k}\right)<\frac{1}{j}$. For each positive integer $n$, put $\delta_{n}:=d\left(x_{n},\left\{x_{k}: k \neq n\right\}\right) \in(0,1)$.

Define $f:\left\{x_{n}: n \in \mathbb{N}\right\} \rightarrow(0, \infty)$ by $f\left(x_{n}\right)=\delta_{n} / n$. Whenever $n \neq k$, we get 


$$
\left|f\left(x_{n}\right)-f\left(x_{k}\right)\right| \leq \frac{\delta_{n}}{n}+\frac{\delta_{k}}{k} \leq \delta_{n}+\delta_{k} \leq 2 d\left(x_{n}, x_{k}\right)
$$

Thus, $f$ is 2-Lipschitz. Since $\left\{x_{n}: n \in \mathbb{N}\right\}$ is closed, by Lemma 2.2, $f$ has a 2-Lipschitz positive extension $g$ to $X$. However, since each $\mathbb{M}_{j}$ is infinite, $\frac{1}{g}$ is unbounded on $\left\{x_{n}: n \in \mathbb{M}_{j}\right\}$ for each $j \in \mathbb{N}$. Since $\operatorname{diam}_{d}\left(\left\{x_{n}: n \in \mathbb{M}_{j}\right\}\right) \leq \frac{1}{j}$, the function $\frac{1}{g}$ while locally Lipschitz fails to be uniformly locally Lipschitz.

While we can't employ Proposition 3.5(b) here, we note that $U L L(X)$ is nevertheless stable under reciprocation precisely when $U L L(X)=L L(X)[6$, Theorem 3.1]. With cofinal completeness of $\langle X, d\rangle$, we of course then have the uniform density of $U L L(X)$ in the real-valued continuous functions. Conversely, without cofinal completeness, we can find a continuous real-valued function $f$ on $X$ for which the third bullet point above fails [3, Theorem 3.4], and such a function cannot be uniformly approximated by uniformly locally Lipschitz functions.

\section{LiPSCHITZ IN THE SMALL FUNCTIONS}

In this case, necessary and sufficient internal conditions on the metric space $\langle X, d\rangle$ for stability under pointwise product are more subtle than they are for stability under reciprocation. In both cases, they agree with the answers recently obtained for the class of uniformly continuous real-valued functions.

J. Cabello-Sánchez made the initial advance [13]; he showed that the uniformly continuous real-valued functions are stable under pointwise product if and only if each nonempty subset $A$ of $\langle X, d\rangle$ is either Bourbaki bounded or contains an infinite uniformly isolated subset $A_{0}$, that is, $\inf \left\{I_{d}(x): x \in A_{0}\right\}>0$. A year after that, Beer, Garrido and Meroño [9] showed that the Cabello-Sánchez conditions amounted to agreement of the Bourbaki bounded subsets with a different generally larger bornology. A year later, Bouziad and Sukhacheva [12] supplied another interesting internal condition: there exists a Bourbaki bounded subset $B$ such that for each $\varepsilon>0$, there exists $n \in \mathbb{N}$ such that $X \backslash S_{d}^{n}(B, \varepsilon)$ is uniformly isolated. They also came up with two attractive function space characterizations (see also [4]).

As to stability of uniform continuity under reciprocation, this was also settled in [9]: the continuous real-valued functions on $X$ must agree with the uniformly continuous real-valued functions on $X$. The well-studied class of spaces for which this agreement holds are now called either the UC-spaces or the Atsuji spaces (see, e.g, $[1,2,23,27])$. This class of metric spaces includes both the compact metric spaces and the uniformly isolated metric spaces.

Some attractive internal characterizations of UC-spaces are now given. Recall that $\alpha>0$ is called a Lebesgue number for an open cover $\mathcal{V}$ of a metric space $\langle X, d\rangle$ if whenever $A \subseteq X$ satisfies $\operatorname{diam}_{d}(A)<\alpha$, there exists $V \in \mathcal{V}$ with $A \subseteq V$.

- each open cover of $X$ has a Lebesgue number;

- whenever $A$ and $B$ are nonempty disjoint closed subsets of $X$, then $\inf \{d(a, b)$ : $a \in A, b \in B\}>0$;

- whenever $\left\langle x_{n}\right\rangle$ is a sequence in $X$ for which $\lim _{n \rightarrow \infty} I_{d}\left(x_{n}\right)=0$, then $\left\langle x_{n}\right\rangle$ clusters; 
- $X^{\prime}$ is compact and $\forall \varepsilon>0, \exists \delta>0$ such that $x \notin S_{d}\left(X^{\prime}, \varepsilon\right) \Rightarrow I_{d}(x)>\delta$.

It is not true in a Cabello-Sánchez space that we can always find a Bourbaki bounded subset $B$ such that $\forall \varepsilon>0, \exists \delta>0$ such that $x \notin S_{d}(B, \varepsilon) \Rightarrow I_{d}(x)>\delta$ $[9,12]$. An example of a Cabello-Sánchez space that is neither Bourbaki bounded nor a UC-space is $(0,1) \cup \mathbb{N}$ equipped with the usual metric of the line. Each UC-space is cofinally complete [3]; $\mathbb{R}$ equipped with the usual metric is cofinally complete but is not a UC-space.

Our characterization of metric spaces for which $L S(X)$ is stable under pointwise product deliberately tries to parallel the statement of [4, Theorem 4].

Theorem 5.1. Let $\langle X, d\rangle$ be a metric space. The following conditions are equivalent:

(1) each subset of $X$ is either Bourbaki bounded or contains an infinite uniformly isolated subset;

(2) $\forall f \in L S(X), \exists k \in \mathbb{N}$ such that $\{x \in X:|f(x)|>k\}$ is uniformly isolated;

(3) $\forall f \in L S(X), \forall g \in L L(\mathbb{R})$ we have $g \circ f \in L S(X)$;

(4) $L S(X)$ is stable under pointwise product;

(5) whenever $f \in L S(X)$ and $h \in \operatorname{Lip}(X)$ we have $f h \in L S(X)$.

Proof. (1) $\Rightarrow(2)$. If (1) holds, then (2) holds for the larger class of uniformly continuous real-valued functions $[4,12]$.

$(2) \Rightarrow(3)$. Suppose $f \in L S(X)$ and $g \in L L(\mathbb{R})$ are arbitrary. Pick $k$ as guaranteed by condition (2) and then $\delta_{1}>0$ such that whenever $|f(x)|>k$, we have $S_{d}\left(x, \delta_{1}\right)=\{x\}$. By the compactness of $[-k, k], g$ restricted to $[-k, k]$ is Lipschitz and so $g \circ f$ is Lipschitz in the small on $f^{-1}([-k, k])$. This means that there exists $\lambda>0$ and $\delta \in\left(0, \delta_{1}\right)$ such that whenever $d\left(x_{1}, x_{2}\right)<\delta$ and $\left|f\left(x_{i}\right)\right| \leq k$ for $i=1,2$ we have

$$
\left|(g \circ f)\left(x_{1}\right)-(g \circ f)\left(x_{2}\right)\right| \leq \lambda d\left(x_{1}, x_{2}\right) .
$$

Since $\delta<\delta_{1}$, the same estimate holds whenever $\left\{x_{1}, x_{2}\right\} \subseteq X$.

$(3) \Rightarrow(4)$. Simply use $g(t)=t^{2}$ from which $L S(X)$ is stable under squaring.

$(4) \Rightarrow(5)$. This trivial.

$(5) \Rightarrow(1)$. We modify a construction used in the proof of Theorem 4 of [4]. Suppose the Cabello-Sánchez criterion (1) fails. We can find a nonempty subset $A$ that is not Bourbaki bounded and each infinite subset $E$ of $A$ satisfies $\inf _{e \in E} I_{d}(e)=$ 0 . From [5, Theorem 3.3], we can find $f \in L S(X)$ that is unbounded on $A$, and by replacing $f$ by $|f|$ we may assume that $f$ has nonnegative values only. By the uniform continuity of $f$, pick $\delta>0$ such $d(x, w)<\delta \Rightarrow|f(x)-f(w)|<\frac{1}{3}$. Since $\{a \in A: f(a)>1\}$ is infinite and not uniformly isolated, we can find $a_{1} \in A$ and $x_{1} \in X$ such that $f\left(a_{1}\right)>1$ and $0<d\left(a_{1}, x_{1}\right)<\delta$. Similarly, $\{a \in A: f(a)>$ $\left.f\left(a_{1}\right)+1\right\}$ is infinite and not uniformly isolated, and we can find $a_{2} \in A$ and $x_{2} \in X$ with $f\left(a_{2}\right)>f\left(a_{1}\right)+1$ and $0<d\left(a_{2}, x_{2}\right)<\frac{\delta}{2}$. Continuing, for each $k \in \mathbb{N}$ choose $a_{k} \in A$ and $x_{k} \in X$ with $f\left(a_{k+1}\right)>f\left(a_{k}\right)+1$ and $0<d\left(a_{k}, x_{k}\right)<\frac{\delta}{k}$. Obviously $f\left(a_{k}\right)>k$ for each integer $k$, and by the choice of $\delta$, whenever $k \neq n$ we have

$$
\min \left\{d\left(a_{k}, a_{n}\right), d\left(x_{k}, x_{n}\right), d\left(a_{k}, x_{n}\right)\right\} \geq \delta
$$


By construction, the sequence $a_{1}, x_{1}, a_{2}, x_{2}, a_{3}, x_{3} \ldots$ has distinct terms. Write $E$ for the range of the sequence and define $h: E \rightarrow \mathbb{R}$ by $h\left(a_{n}\right)=d\left(a_{n}, x_{n}\right)$ and $h\left(x_{n}\right)=0$. We intend to show that $h$ is 1-Lipschitz on $E$. First, for each $n \in \mathbb{N}$,

$$
\frac{\left|h\left(a_{n}\right)-h\left(x_{n}\right)\right|}{d\left(a_{n}, x_{n}\right)}=\frac{d\left(a_{n}, x_{n}\right)}{d\left(a_{n}, x_{n}\right)}=1
$$

and if $n \neq k$ we have $\frac{\left|h\left(x_{n}\right)-h\left(x_{k}\right)\right|}{d\left(x_{n}, x_{k}\right)}=0<1$. Also, if $n \neq k$ we have

$$
\frac{\left|h\left(a_{n}\right)-h\left(x_{k}\right)\right|}{d\left(a_{n}, x_{k}\right)}=\frac{d\left(a_{n}, x_{n}\right)}{d\left(a_{n}, x_{k}\right)}<\frac{\delta / n}{\delta} \leq 1,
$$

Finally, if $k<n$, we obtain

$$
\frac{\left|h\left(a_{n}\right)-h\left(a_{k}\right)\right|}{d\left(a_{n}, a_{k}\right)}<\frac{\delta / k}{\delta} \leq 1
$$

These estimates taken together verify that $h$ is 1-Lipschitz on $E$. We can extend $h$ to a nonnegative 1-Lipschitz function on $X$ [20, pp. 43-44], and to avoid extra notation, we use $h$ to denote this extension as well. Since $f\left(a_{n}\right)>n$ for each $n \in \mathbb{N}$, we obtain for each $n \in \mathbb{N}$

$$
\left|f\left(a_{n}\right) h\left(a_{n}\right)-f\left(x_{n}\right) h\left(x_{n}\right)\right|=f\left(a_{n}\right) h\left(a_{n}\right)>n d\left(a_{n}, x_{n}\right) .
$$

Since $a_{n}$ and $x_{n}$ can be made arbitrarily close together, the pointwise product $f h$ cannot belong to $L S(X)$ and so condition (5) fails.

Moving to reciprocation for $L S(X)$, we need a fact about this vector lattice observed in [16]: a bounded Lipschitz in the small real-valued function defined on a nonempty subset $A$ of $X$ is already Lipschitz on the subset $A$. Letting $\delta$ be the distance control parameter and $\lambda$ the local Lipschitz constant and choosing $\alpha$ with $\sup \left\{\left|f\left(a_{1}\right)-f\left(a_{2}\right)\right|: a_{1}, a_{2} \in A\right\}<\alpha$, it is each to check that $\max \left\{\lambda, \frac{\alpha}{\delta}\right\}$ is a Lipschitz constant for $f$ on $A$.

Theorem 5.2. Let $\langle X, d\rangle$ be a metric space. The following conditions are equivalent:

(1) the metric space is a UC-space;

(2) $L S(X)$ is stable under reciprocation;

(3) whenever $g \in \operatorname{Lip}(X)$ is non-vanishing, then $\frac{1}{g} \in L S(X)$.

Proof. $(1) \Rightarrow(2)$. If $\langle X, d\rangle$ is a UC-space, then $L S(X)=L L(X)$ [6, Theorem 3.3].

$(2) \Rightarrow(3)$. This is trivial.

$(3) \Rightarrow(1)$. If condition (3) holds and $X$ is not complete, take $p$ in $\hat{X} \backslash X$ where $\langle\hat{X}, \hat{d}\rangle$ is the completion of $X$. While $x \mapsto \hat{d}(x, p)$ is a Lipschitz function on $X$, its reciprocal is not Lipschitz in the small or even uniformly locally Lipschitz because it fails to be uniformly locally bounded.

Now consider the possibility that $\langle X, d\rangle$ while complete is not a UC-space. We can find sequences $\left\langle x_{n}\right\rangle$ and $\left\langle w_{n}\right\rangle$ such that for each $n, 0<d\left(x_{n}, w_{n}\right)<\frac{1}{n}$ but such that $\left\langle x_{n}\right\rangle$ fails to cluster. By completeness, $\left\langle x_{n}\right\rangle$ has no Cauchy subsequence 
and so $\left\{x_{n}: n \in \mathbb{N}\right\}$ is not totally bounded. By passing to a subsequence, we may assume that for some $\delta \in(0,1)$

(a) whenever $n \neq j$, we have $d\left(x_{n}, x_{j}\right)>\delta$, and

(b) for each $n \in \mathbb{N}, d\left(x_{n}, w_{n}\right)<\frac{\delta}{3}$.

Next put $A:=\left\{x_{n}: n \in \mathbb{N}\right\} \cup\left\{w_{n}: n \in \mathbb{N}\right\}$. By conditions (a) and (b), if $\left\{a_{1}, a_{2}\right\} \subseteq A$ with $0<d\left(a_{1}, a_{2}\right)<\frac{\delta}{3}$, then for some $n \in \mathbb{N},\left\{a_{1}, a_{2}\right\}=\left\{x_{n}, w_{n}\right\}$.

Now define $f: A \rightarrow(0, \infty)$ by $f\left(x_{n}\right)=d\left(x_{n}, w_{n}\right)^{2}$ and $f\left(w_{n}\right)=d\left(x_{n}, w_{n}\right)$. Then if $d\left(a_{1}, a_{2}\right)<\frac{\delta}{3}$, we have $\left|f\left(a_{1}\right)-f\left(a_{2}\right)\right| \leq d\left(a_{1}, a_{2}\right)$ because for each $n \in \mathbb{N}$

$$
\left|f\left(x_{n}\right)-f\left(w_{n}\right)\right|=\left|d\left(x_{n}, w_{n}\right)-1\right| d\left(x_{n}, w_{n}\right)<d\left(x_{n}, w_{n}\right) .
$$

As $f$ is Lipschitz in the small on $A$ and $f$ is bounded on $A, f$ is actually Lipschitz on $A$. By Lemma 2.2, $f$ has a positive Lipschitz extension $g$ to $X$. But $\frac{1}{g} \notin L S(X)$ because

$$
\frac{\left|\frac{1}{g}\left(x_{n}\right)-\frac{1}{g}\left(w_{n}\right)\right|}{d\left(x_{n}, w_{n}\right)}=\frac{1-d\left(x_{n}, w_{n}\right)}{d\left(x_{n}, w_{n}\right)^{3}}>\frac{2 / 3}{d\left(x_{n}, w_{n}\right)^{3}}
$$

while $\lim _{n \rightarrow \infty} d\left(x_{n}, w_{n}\right)=0$.

As might now be anticipated, our conditions on $\langle X, d\rangle$ that characterize when $L S(X)$ is stable under reciprocation are exactly those that are necessary and sufficient for $L S(X)=L L(X)$ [6, Theorem 3.3].

\section{Extensions of Cauchy-Lipschitz functions and Lipschitz in the SMALL FUNCTIONS}

In his seminal article on Cauchy continuous functions [31], Snipes proved that (1) a Cauchy continuous function defined on a nonempty subset $A$ of a metric space $\langle X, d\rangle$ into a complete metric space has a Cauchy continuous extension to $\operatorname{cl}(A)$, and $(2)$ if $\langle X, d\rangle$ is a complete metric space, then the Cauchy continuous functions on $\langle X, d\rangle$ agree with the continuous functions on $\langle X, d\rangle$ whatever the metric target space may be. From this it follows that each real-valued Cauchy continuous function $f$ on a nonempty subset $A$ of an arbitrary metric space $\langle X, d\rangle$ has a Cauchy continuous extension to $X$. We sketch the argument: (a) extend $f$ to a continuous function on the closure of $A$ relative to the completion $\langle\hat{X}, \hat{d}\rangle$ of $\langle X, d\rangle$; (b) extend this initial extension to a continuous function on the completion using the Tietze extension theorem; this second extension must be Cauchy continuous by completeness of $\hat{d}$; (c) restrict this second extension back down to $X$.

We begin this section by showing that analogous results hold for Cauchy-Lipschitz functions.

Theorem 6.1. Let $A$ be a nonempty subset of $\langle X, d\rangle$ and let $\langle Y, \rho\rangle$ be a complete metric space. Suppose $f: A \rightarrow Y$ is a Cauchy-Lipschitz function. Then $f$ has a Cauchy-Lipschitz extension to $\operatorname{cl}(A)$.

Proof. Since $f$ is Cauchy continuous, by [31, Theorem 7] let $\tilde{f}$ be a Cauchy continuous extension of $f$ to $\operatorname{cl}(A)$. We intend to show that $\tilde{f}$ is Lipschitz when restricted 
to the range of each Cauchy sequence $\left\langle x_{n}\right\rangle$ in $\operatorname{cl}(A)$. For each $n \in \mathbb{N}$ let $\left\langle x_{n}^{k}\right\rangle_{k=1}^{\infty}$ be a sequence in $A$ such that for all $n$ and $k, d\left(x_{n}^{k}, x_{n}\right)<\frac{1}{n k}$. We claim that the set $B:=\left\{x_{n}^{k}: n, k \in \mathbb{N}\right\}$ is totally bounded.

To see this, given $\varepsilon>0$, there is an integer $m>\frac{2}{\varepsilon}$ such that $d\left(x_{n}, x_{m}\right)<\frac{\varepsilon}{2}$ whenever $n \geq m$. We also have

$$
d\left(x_{n}, x_{n}^{k}\right)<\frac{1}{n k} \leq \frac{1}{m}
$$

whenever $n \geq m$. It follows from the triangle inequality that $\left\{x_{n}^{k}: n \geq m\right.$ and $k \in$ $\mathbb{N}\} \subseteq S_{d}\left(x_{m}, \varepsilon\right)$. In addition, we have $d\left(x_{n}^{k}, x_{n}\right)<\varepsilon$ whatever $n$ may be if $k>\frac{1}{\varepsilon}$. From these estimates,

$$
\left\{x_{n}^{k}: n<m \text { and } k>\frac{1}{\varepsilon}\right\} \subseteq \cup_{n<m} S_{d}\left(x_{n}, \varepsilon\right) .
$$

As only finitely many $x_{n}^{k}$ are unaccounted for, $B$ can be covered with a finite number of open balls of radius $\varepsilon$, and the claim holds.

Since $B \subseteq A$ and $f$ is Cauchy-Lipschitz on $A,\left.\tilde{f}\right|_{B}=\left.f\right|_{B}$ is Lipschitz [7, Proposition 3.4]. Let $\lambda>0$ be a Lipschitz constant for $\left.\tilde{f}\right|_{B}$. Whenever $n, m, k$ are positive integers, we have

$$
\begin{aligned}
\rho\left(\tilde{f}\left(x_{n}\right), \tilde{f}\left(x_{m}\right)\right) & \leq \rho\left(\tilde{f}\left(x_{n}\right), \tilde{f}\left(x_{n}^{k}\right)\right)+\rho\left(\tilde{f}\left(x_{n}^{k}\right), \tilde{f}\left(x_{m}^{k}\right)\right)+\rho\left(\tilde{f}\left(x_{m}^{k}\right), \tilde{f}\left(x_{m}\right)\right) \\
& \leq \rho\left(\tilde{f}\left(x_{n}\right), \tilde{f}\left(x_{n}^{k}\right)\right)+\lambda d\left(x_{n}^{k}, x_{m}^{k}\right)+\rho\left(\tilde{f}\left(x_{m}^{k}\right), \tilde{f}\left(x_{m}\right)\right) .
\end{aligned}
$$

Letting $k \rightarrow \infty$, the continuity of $\tilde{f}$ yields $\rho\left(\tilde{f}\left(x_{n}\right), \tilde{f}\left(x_{m}\right)\right) \leq \lambda d\left(x_{n}, x_{m}\right)$ as required.

For our general extension theorem for real-valued Cauchy-Lipschitz functions, in lieu of Tietze's theorem, we use this result of Czipszer and Gehér [14, 15]: each real-valued locally Lipschitz function defined on a closed subset of a metric space has a locally Lipschitz extension to the entire space.

Theorem 6.2. Let $A$ be a nonempty subset of a metric space $\langle X, d\rangle$ and let $f \in$ $C L(A)$. Then there exists $g \in C L(X)$ that extends $f$.

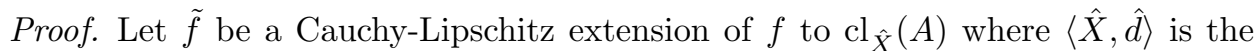
completion of the initial metric space. By the Czipszer-Gehér result and [7, Proposition 3.2], $\tilde{f}$ can be extended to a locally Lipschitz function $\hat{g}$ on $\langle\hat{X}, \hat{d}\rangle$. By the completeness of $\hat{d}$, the second extension is Cauchy-Lipschitz [7, Theorem 3.5]. Finally, put $g:=\left.\hat{g}\right|_{X}$.

Since extension theorems for locally Lipschitz functions seem to parallel those for continuous functions, and extension theorems for Cauchy-Lipschitz functions seem to parallel those for Cauchy continuous functions, one would expect that extension theorems for Lipschitz in the small functions would parallel those for uniformly continuous functions. We focus on two results. First, there is a result that can be found in introductory analysis texts: a uniformly continuous function on a nonempty subset $A$ of $\langle X, d\rangle$ to a complete metric space $\langle Y, \rho\rangle$ has a uniformly continuous extension to $\operatorname{cl}(A)$. Second, we have a less elementary result that falls out of [17, Theorem 7]: a metric space $\langle X, d\rangle$ is small determined if and only if each 
uniformly continuous real-valued function on $X$ can be extended to a uniformly continuous function on each metric space in which $\langle X, d\rangle$ is isometrically embedded.

Theorem 6.3. Let $A$ be a nonempty subset of $\langle X, d\rangle$ and let $\langle Y, \rho\rangle$ be a complete metric space. Suppose $f: A \rightarrow Y$ is Lipschitz in the small. Then $f$ has a Lipschitz in the small extension to $\operatorname{cl}(A)$ that has the same distance control and the same uniform local Lipschitz constant.

Proof. Suppose whenever $d\left(a_{1}, a_{2}\right)<\delta$ in $A$, we have $\rho\left(f\left(a_{1}\right), f\left(a_{2}\right)\right) \leq \lambda d\left(a_{1}, a_{2}\right)$. Extend $f$ to a uniformly continuous function $\tilde{f}$ on $\operatorname{cl}(A)$, and let $x$ and $w$ be distinct points of the closure with $d(x, w)<\delta$. We can find sequences $\left\langle a_{x}^{k}\right\rangle$ and $\left\langle a_{w}^{k}\right\rangle$ in $A$ convergent to $x$ and $w$, respectively such that for each $k \in \mathbb{N}$, we have $d\left(a_{x}^{k}, a_{w}^{k}\right)<\delta$. In view of the inequality

$$
\rho(\tilde{f}(x), \tilde{f}(w)) \leq \rho\left(\tilde{f}(x), f\left(a_{x}^{k}\right)\right)+\lambda d\left(a_{x}^{k}, a_{w}^{k}\right)+\rho\left(f\left(a_{w}^{k}\right), \tilde{f}(w)\right)
$$

the assertion of our theorem follows from the continuity of $\tilde{f}$.

We now introduce the modulus of continuity function $\omega_{f}:[0, \infty) \mapsto[0, \infty][10]$ associated with a function $f$ between metric spaces $\langle X, d\rangle$ and $\langle Y, \rho\rangle$ :

$$
\omega_{f}(t):=\sup \{\rho(f(x), f(w)): x, w \in X \text { and } d(x, w) \leq t\} \quad(t \geq 0) .
$$

Some obvious properties of $\omega_{f}$ are now listed as a courtesy to the reader:

- $\omega_{f}(0)=0$ and $\omega_{f}$ is nondecreasing on $[0, \infty)$;

- $f$ is uniformly continuous if and only if $\omega_{f}$ is continuous at $t=0$;

- $f$ is $\lambda$-Lipschitz if and only if $t \mapsto \lambda t$ majorizes $\omega_{f}$;

- $f$ is Lipschitz in the small with distance control $\delta$ and uniform local Lipschitz constant $\lambda$ if and only if $t \rightarrow \lambda t$ majorizes $\omega_{f}$ on $[0, \delta)$.

We next give necessary and sufficient conditions for a given Lipschitz in the small function to be Lipschitz in terms of its modulus of continuity. Recall that a real-valued function defined on a convex subset $C$ of a normed linear space is called concave if whenever $\left\{c_{1}, c_{2}\right\} \subseteq C$ and $\alpha \in[0,1]$, we have $\alpha f\left(c_{1}\right)+(1-\alpha) f\left(c_{2}\right) \leq$ $f\left(\alpha c_{1}+(1-\alpha) c_{2}\right)$.

Proposition 6.4. Let $\langle X, d\rangle$ and $\langle Y, \rho\rangle$ be metric spaces and suppose $f: X \rightarrow Y$ is Lipschitz in the small. The following conditions are equivalent:

(1) $\omega_{f}$ has a concave majorant;

(2) $\omega_{f}$ has an affine majorant, that is, one of the form $t \mapsto \alpha t+\beta$;

(3) there exists scalars $\alpha$ and $\beta$ and $n \in \mathbb{N}$ such that $\forall t \geq n, \omega_{f}(t) \leq \alpha t+\beta$;

(4) $f$ is Lipschitz.

Proof. Note that $(2) \Rightarrow(3)$ and (4) $\Rightarrow(1)$ are trivial, since $t \mapsto \lambda t$ is a concave function.

$(1) \Rightarrow(2)$. Let $g$ be a concave majorant of $\omega_{f}$. Now a concave function on $[0, \infty)$ is differentiable on $(0, \infty)$ except at most countably many points, and at points of differentiability, the associated tangent line to the graph supports the hypograph of the function (see, e.g., [29, pp. 5-13]); taking $t_{0} \in(0, \infty)$ where $g^{\prime}\left(t_{0}\right)$ exists, this means

$$
w_{f}(t) \leq g(t) \leq g\left(t_{0}\right)+g^{\prime}\left(t_{0}\right)\left(t-t_{0}\right) \quad(t \geq 0) .
$$


STABILITY OF LIPSCHITZ-TYPE FUNCTIONS UNDER POINTWISE PRODUCT AND RECIPROCATIOII

(3) $\Rightarrow(4)$. Note that $\alpha \geq 0$ since $\alpha t+\beta \geq 0$ for all $t \geq n$. One can assume that $\beta \geq \omega_{f}(n)$ so that $t \mapsto \alpha t+\beta$ majorizes the modulus of continuity throughout $[0, \infty)$ because $\omega_{f}$ is nondecreasing. Let $\delta>0$ and $\lambda>0$ be such that $\omega_{f}(t) \leq \lambda t$ if $t \in[0, \delta)$. By replacing $\beta$ by a larger value if needed, we may assume that $\lambda \leq \frac{\alpha \delta+\beta}{\delta}:=\mu$. We claim that $\forall t \geq 0, \omega_{f}(t) \leq \mu t$. This is clear if $t \in[0, \delta)$ because $\lambda \leq \mu$. On the other hand, if $t \geq \delta$, then

$$
\omega_{f}(t) \leq \alpha t+\beta \leq \alpha t+\beta \frac{t}{\delta}=\mu t
$$

This shows that $f$ is $\mu$-Lipschitz.

Theorem 6.5. Let $\langle X, d\rangle$ be a metric space. The following conditions are equivalent:

(1) $\langle X, d\rangle$ is small determined;

(2) for each $f \in L S(X)$, its modulus of continuity has an affine majorant;

(3) each member of $L S(X)$ has a Lipschitz in the small extension to any metric space in which $X$ is isometrically embedded;

(4) there exists a normed linear space $\langle W,\|\cdot\|\rangle$ isometrically containing $\langle X, d\rangle$ for which each member of $L S(X)$ can be extended to a member of $L S(W)$.

Proof. For $(1) \Rightarrow(2)$, if $\langle X, d\rangle$ is small determined, then by Proposition 6.4 in particular, for each real-valued Lipschitz in the small function $f$ on $X, \omega_{f}$ has an affine majorant. For $(2) \Rightarrow(3)$, if $f \in L S(X)$, then by condition (2) and Proposition 6.4, we have $f \in \operatorname{Lip}(X)$, and so $f$ actually has a Lipschitz extension to any metric space in which $X$ is isometrically embedded $[20$, p. 43]. The implication $(3) \Rightarrow(4)$ follows from the fact that each metric space can be isometrically embedded in some normed linear space, e.g., the bounded continuous real-valued functions on the space equipped with the supremum norm. For $(4) \Rightarrow(1)$, it suffices to show that each real-valued Lipschitz in the small function on $X$ is Lipschitz [17, 24]. Let $f \in L S(X)$; by condition (4), $\exists g \in L S(W)$ extending $f$. But by its convexity, $\langle W,\|\cdot\|\rangle$ is a small determined space, whence $g$ is Lipschitz, and so $f$ is Lipschitz as well.

We now use Proposition 6.4 to derive a folk-theorem alluded to in the Wikipedia entry on the modulus of continuity. We know of no explicit reference for this result in the actual literature, but it certainly is not new.

Theorem 6.6. Suppose $f$ is uniformly continuous and real-valued on the metric space $\langle X, d\rangle$. The following conditions are equivalent:

(1) $f$ can be uniformly approximated by elements of Lip $(X)$;

(2) there exists $g \in \operatorname{Lip}(X)$ at a finite uniform distance from $f$;

(3) $\omega_{f}$ has an affine majorant.

Proof. (1) $\Rightarrow(2)$ is trivial. For $(2) \Rightarrow(3)$, choose $\nu>0$ such that

$$
\sup _{x \in X}|g(x)-f(x)|<\nu \text {. }
$$

It is clear that for each $t \geq 0,\left|\omega_{g}(t)-\omega_{f}(t)\right| \leq 2 \nu$. Now if $\lambda$ is a Lipschitz constant for $g$, then $t \mapsto \lambda t$ majorizes $\omega_{g}$, whence $t \mapsto \lambda t+2 \nu$ majorizes $w_{f}$. For $(3) \Rightarrow(1)$, let $\varepsilon>0$ be arbitrary. By the uniform continuity of $f$ we can find $h \in L S(X)$ with $\sup _{x \in X}|h(x)-f(x)|<\varepsilon[6,17]$. If $t \mapsto \alpha t+\beta$ majorizes $\omega_{f}$, then $t \mapsto \alpha t+\beta+2 \varepsilon$ 
majorizes $\omega_{h}$. By Proposition $6.4, h$ is Lipschitz and uniformly approximates $f$ within $\varepsilon$.

We remark that with some work, $(3) \Rightarrow(1)$ of our last theorem can be obtained from [10, Proposition 2.1], where the desired subadditive function is the concave envelope of $\omega_{f}$. We leave this to the imagination of the interested reader.

Our final result of this article pulls together some of our machinery in an intriguing way. We would not be surprised if the equivalence of conditions (1) and (2) has already been observed.

Theorem 6.7. Let $\langle X, d\rangle$ be a metric space. Then following conditions are equivalent:

(1) $\langle X, d\rangle$ is compact;

(2) each real-valued continuous function on $X$ can be uniformly approximated by members of $\operatorname{Lip}(X)$;

(3) $\langle X, d\rangle$ is both a UC-space and small determined.

Proof. If $X$ is compact, then $\operatorname{Lip}(X)=L L(X)$ [30], and $L L(X)$ is uniformly dense in the real-valued continuous functions with no assumptions on $X[14,16,26]$. If $\operatorname{Lip}(X)$ is uniformly dense in the real-valued continuous functions, then clearly each continuous real-valued function is uniformly continuous. On the other hand, by Theorem 6.6, for each $f \in L S(X), \omega_{f}$ has an affine majorant, so that by Theorem $6.4, L S(X) \subseteq \operatorname{Lip}(X)$, and $\langle X, d\rangle$ is small determined.

Finally suppose $\langle X, d\rangle$ is both a UC-space and small determined. By UC-ness and Theorem 5.2, $L S(X)$ is stable under reciprocation, and so $\operatorname{Lip}(X)$ is stable under reciprocation. By Theorem 3.4, we conclude that the metric space is compact.

\section{REFERENCES}

1. M. Atsuji, Uniform continuity of continuous functions of metric spaces, Pacific J. Math. 8 (1958), 11-16.

2. G. Beer, Topologies on closed and closed convex sets, Kluwer Academic Publishers, Dordrecht, Holland, 1993.

3. G. Beer, Between compactness and completeness, Top. Appl. 155 (2008), 503-514.

4. G. Beer, McShane's extension theorem revisited, to appear, Vietnam J. Math.

5. G. Beer and M. I. Garrido, Bornologies and locally Lipschitz functions, Bull. Aust. Math. Soc. 90 (2014), 257-263.

6. G. Beer and M. I. Garrido, Locally Lipschitz functions, cofinal completeness, and UC spaces, J. Math. Anal. Appl. 428 (2015), 804-816.

7. G. Beer and M. I. Garrido, On the uniform approximation of Cauchy continuous functions, Top. Appl. 208 (2016), 1-9.

8. G. Beer and M. I. Garrido, Real-valued Lipschitz functions and metric properties of functions, to appear, J. Math. Anal. Appl. 486 (2020).

9. G. Beer, M. I. Garrido, and A. S. Meroño, Uniform continuity and a new bornology for a metric space, Set-Valued Var. Anal. 26 (2018), 49-65.

10. Y. Benyamini and J. Lindenstrauss, Geometric nonlinear functional analysis vol. 1, American Math. Soc., Providence, 1998.

11. J. Borsik, Mappings preserving Cauchy sequences, Časopis pěst. Mat. 113 (1988), 280-285.

12. A. Bouziad and E. Sukhacheva, Preservation of uniform continuity under pointwise product, Top. Appl. 254 (2019), 132-144.

13. J. Cabello-Sánchez, $U(X)$ as a ring for metric spaces $X$, Filomat 31 (2017), 1981-1984.

14. Ş. Cobzaş, R. Miculescu, and A. Nicolae, Lipschitz functions, Springer LNM 2241, Cham, Switzerland, 2019.

15. J. Czipszer and L. Gehér, Extensions of functions satisfying a Lipschitz condition, Acta Math. Acad. Sci. Hung. 6 (1955), 213-220. 
STABILITY OF LIPSCHITZ-TYPE FUNCTIONS UNDER POINTWISE PRODUCT AND RECIPROCATIOIY

16. M. I. Garrido and J. Jaramillo, Homomorphisms on function lattices, Monatsh. Math. 141 (2004), 127-146.

17. M. I. Garrido and J. Jaramillo, Lipschitz-type functions on metric spaces, J. Math. Anal. Appl. 340 (2008), 282-290.

18. M. I. Garrido and A.S. Meroño, New types of completeness in metric spaces, Ann. Acad. Sci. Fenn. Math. 39 (2014), 733-758.

19. J. Hejcman, Boundedness in uniform spaces and topological groups, Czech Math. J. 9 (1959), 544-563.

20. J. Heinonen, Lectures on analysis on metric spaces, Springer, New York, 2001.

21. A. Hohti, On uniform paracompactness, Ann. Acad. Sci. Fenn. Series A Math. Diss. 36 (1981), $1-46$.

22. N. Howes, Modern analysis and topology, Springer, New York, 1995.

23. T. Jain and S. Kundu, Atsuji spaces: equivalent conditions, Top. Proc. 30 (2006), 301-325.

24. D. Leung and W.-K. Tang, Functions that are Lipschitz in the small, Rev. Mat. Complut. 30 (2017), 25-34.

25. J. Luukkainen, Rings of functions in Lipschitz topology, Ann. Acad. Sci. Fenn. Series A. I. Math. 4 (1978-79), 119-135.

26. R. Miculescu, Approximation of continuous functions by Lipschitz functions, Real Anal. Exhange 26 (2000-2001), 449-452.

27. A. A. Monteiro and M. M. Peixoto, Le nombre de Lebesgue et la continuité uniform, Portugaliae Math. 10 (1951), 105-113.

28. M. Rice, A note on uniform paracompactness, Proc. Amer. Math. Soc. 62 (1977), 359-362.

29. A. W. Roberts and D. Varberg, Convex functions, Academic Press, New York, 1973.

30. C. Scanlon, Rings of functions with certain Lipschitz properties, Pacific J. Math. 32 (1970), 197-201.

31. R. Snipes, Functions that preserve Cauchy sequences, Nieuw Archief Voor Wiskunde 25 (1977), 409-422.

32. S. Willard, General Topology, Addison-Wesley, Reading, MA, 1970.

Department of Mathematics, California State University los Angeles, 5151 State University Drive, Los Angeles, CAlifornia 90032, USA

Email address: gbeer@cslanet.calstatela.edu

Department of Mathematical Sciences, Kent State University, Kent, Ohio 44242, USA

Email address: lgarcial@kent.edu

Instituto de Matemática Interdisciplinar (IMI), Departamento de Álgebra, Geometría y Topología, Universidad Complutense de Madrid, 28040 Madrid, Spain

Email address: maigarri@mat.ucm.es 